\title{
Thermal Radiation from Isolated Neutron Stars
}

\author{
G. G. Pavlov \\ The Pennsylvania State University, Department of Astronomy and \\ Astrophysics, 525 Davey Lab, University Park, PA 16802, U.S.A. \\ V. E. Zavlin \\ Max-Planck Institut für Extraterrestrische Physik, 85740 Garching, \\ Germany
}

\begin{abstract}
The analysis of thermal radiation emitted from the atmospheres of isolated neutron stars allows one to measure their surface temperatures, magnetic fields, masses, and radii, as well as the chemical composition of their atmospheres. Thus, multiwavelength observations of this radiation provide an important tool for studying the structure and evolution of neutron stars and for elucidating properties of the superdense matter in their interiors. We describe recent theoretical and observational results on thermal radiation from radio pulsars and radio-quiet neutron stars and discuss their astrophysical implications.
\end{abstract}

\section{Introduction}

Our knowledge about properties and evolution of usual stars is based upon the analysis of thermal radiation emitted from their atmospheres. Neutron stars (NSs) were discovered only 32 years ago as radio pulsars, and observational study of their extremely faint thermal radiation was virtually impossible until the first X-ray and optical space observatories were launched. Thermal radiation has been observed with these observatories from a number of isolated NSs, including radio pulsars and radio-quiet NSs, in soft X-ray and UV-optical ranges. Studying this radiation provides unique information on the NS surface temperature, magnetic field, gravitational acceleration, and chemical composition. The analysis of these properties allows one to trace the thermal evolution of NSs (Tsuruta 1998) and constrain still poorly-known properties of the superdense matter in the NS interiors (Pethick 1992; Van Riper, Link, \& Epstein 1995; Yakovlev, Levenfish, \& Shibanov 1999).

It is well-known that observational manifestations of NSs are very diverse and that these stars are seen in a very broad energy range from radio to gammarays. From which objects and at which energies can one observe the NS thermal radiation? Very young, active pulsars (e.g., the Crab pulsar) are very hot, with expected surface temperatures $\sim 1-2 \mathrm{MK}$, but their nonthermal radiation dominates in any energy range, i.e., their thermal radiation is hardly observable (Becker \& Trümper 1997). In middle-aged pulsars $\left(10^{4}-10^{6} \mathrm{yr}\right)$, with temperatures $\sim 0.3-1 \mathrm{MK}$, the nonthermal component is much fainter, and thermal 
radiation can dominate in the soft $\mathrm{X}$-ray and UV ranges. So far, thermal Xrays have been observed from the middle-aged pulsars B0656+14, J0633+1746 (Geminga), B1055-52, and B0833-45 (the Vela pulsar)-see Ögelman (1995), Halpern \& Wang (1997), and Zavlin \& Pavlov (2000). The soft components of their X-ray spectra are emitted from the entire visible NS surface. The hard components possibly originate from the pulsar's polar caps' heating by relativistic particles produced in the NS magnetosphere. Both the soft and hard components are pulsed with typical pulsed fractions of $\sim 10 \%-30 \%$. The detected UV-optical radiation from these pulsars is mainly nonthermal, but the thermal component is expected to dominate at $\lambda \lesssim 2000-3000 \AA$ (e.g., Pavlov, Welty, \& Còrdova 1997; Mignani, Caraveo, \& Bignami 1998). The surfaces of old pulsars are too cold to be seen in X-rays, but their polar caps are expected to be hot enough, up to a few MK. Pulsed radiation from polar caps was probably detected from the nearby old pulsars B0950+08 and B1929+10 (Yancopoulos, Hamilton, \& Helfand 1994; Wang \& Halpern 1997) and from the millisecond pulsar J0437-4715 (Zavlin \& Pavlov 1998), although additional observations are needed to prove the thermal nature of this radiation. Thermal radiation from the colder surfaces of old pulsars, with temperatures $\lesssim 0.1 \mathrm{MK}$, is observable in the optical-UV range (Pavlov, Stringfellow, \& Còrdova 1996a).

In addition to active pulsars, a number of radio-quiet NSs emitting thermallike X-rays have been discovered, with typical temperatures $\sim 0.5-5 \mathrm{MK}$ (see Caraveo, Bignami, \& Trümper 1996 for a review and references). The hottest objects of this class are found in central regions of supernova remnants (e.g., RX J0822-4300 in Puppis A-Zavlin, Trümper, \& Pavlov 1999). Colder and, apparently, older objects have been detected in both X-ray and optical ranges (e.g., RX J1856+3754-Walter \& Matthews 1997).

Thermal radiation from the NS surface probably has been observed from Type I, bursting NS transients (e.g., Aql X-1, Cen X-4) in quiescence (Rutledge et al. 1999). The surfaces of these NSs are heated up to 1-2 MK by thermonuclear burning of the matter accreted during the outbursts (Brown, Bildsten, \& Rutledge 1998), so that the radiation of these old NSs in quiescence resembles that of young, isolated, cooling NSs.

Thus, the NS surface layers (atmospheres) have been directly observed in about a dozen of objects. Much more detailed data on thermal radiation from these and other NSs are expected from the recently launched X-ray observatories Chandra and XMM. To interpret these observations, detailed and accurate models for the spectra and light curves of thermal radiation from NSs are needed.

\section{Modeling of the Neutron Star Atmospheres}

Properties of the NS thermal radiation are determined, as in usual stars, by assuming a thin, partially ionized atmosphere with temperature growing inward. As a result, the NS thermal radiation may be substantially different from the blackbody radiation (Pavlov \& Shibanov 1978). Modeling of the NS atmospheres requires a special approach because NSs may possess very strong magnetic fields, $B \sim 10^{11}-10^{13} \mathrm{G}$. In such fields, the electron cyclotron energy, $E_{c e}=11.6\left(B / 1 \times 10^{12} \mathrm{G}\right) \mathrm{keV}$, strongly exceeds the thermal energy, $k T \sim 0.01-1 \mathrm{keV}$. As a result, the atmospheres are essentially anisotropic, so 
that absorption and emission of photons depend on the direction of the photon wave vector, and the radiation propagates there as two normal (polarization) modes with nearly orthogonal polarizations and quite different opacities (Gnedin \& Pavlov 1974; Bulik \& Pavlov 1996). The energy dependences of these opacities are substantially different from each other and from the opacity at $B=0$. Since the ratio of the cyclotron energy to the Coulomb energy, $\beta=E_{c e} /\left(Z^{2} \mathrm{Ry}\right)=850 Z^{-2}\left(B / 1 \times 10^{12} \mathrm{G}\right)$, is very large, the structure of atoms and ions is distorted by the strong magnetic field, which changes the energies and strengths of spectral features and ionization equilibrium of the atmospheric plasma. As a result, the spectrum, angular distribution, and polarization of thermal radiation depend on the magnetic field. Another important effect is that the nonuniform magnetic field leads to a nonuniform surface temperature distribution because of anisotropic heat conduction (Greenstein \& Hartke 1983; Shibanov \& Yakovlev 1996), which enhances pulsations of thermal radiation due to NS rotation.

The high density of the atmospheric matter $\left(\sim 1-100 \mathrm{~g} \mathrm{~cm}^{-3}\right.$ at unit optical depth) caused by the immense gravitational acceleration, $g \sim 10^{14}-10^{15} \mathrm{~cm} \mathrm{~s}^{-2}$, poses additional complications. In particular, the nonideality (pressure) effects lead to pressure ionization and smooth out the spectral dependences of the opacities. The huge surface gravity leads to chemical stratification of NS atmospheres, so that upper layers, which determine the properties of the emitted radiation, are comprised of the lightest element present. This means, in particular, that if a NS has accreted some amount of hydrogen (e.g., from the circumstellar medium or from the envelope ejected during the supernova explosion), its radiative properties are determined by the hydrogen atmosphere.

A convenient approach to modeling of NS atmospheres was described by Pavlov et al. (1995). It includes, as for usual stars, solving a set of equations for hydrostatic equilibrium, energy balance, ionization equilibrium, and radiative transfer, complemented by calculations of spectral opacities for a partially ionized, nonideal plasma. For atmospheres with strong magnetic fields, two coupled equations of radiative transfer for the intensities of two polarization modes are to be solved. The input parameters for the modeling are the chemical composition, effective temperature $T_{\text {eff }}$ (or total radiative flux $\propto T_{\text {eff }}^{4}$ ), magnetic field $B$ (including the field orientation at the radiating NS surface), and gravitational acceleration $g$ (or the NS mass $M$ and radius $R$ ).

\subsection{Low-field Neutron Star Atmospheres}

It is commonly accepted that very old NSs (e.g., millisecond pulsars) have "low" surface magnetic fields, $B \sim 10^{8}-10^{9} \mathrm{G}$, which do not affect the X-ray opacities of the atmospheric plasma at temperatures of interest (at $E_{c e} \ll E, E_{c e} \ll k T$, and $\beta \ll 1$. The first models of low-field NS atmospheres were calculated by Romani (1987). Further works (Rajagopal \& Romani 1996; Zavlin, Pavlov, \& Shibanov 1996) used improved opacities (Iglesias \& Rogers 1996) for pure hydrogen, helium, and iron compositions. These works have shown that the spectra of radiation emerging from a light-element $(\mathrm{H}$ or $\mathrm{He})$ atmosphere are much harder (less steep) than the blackbody spectra at $E \gtrsim k T_{\text {eff }}$ (see Figure 1). The reason for such behavior is that the hydrogen and helium opacities decrease with increasing $E$, so that the radiation of higher energies is formed in deeper and 


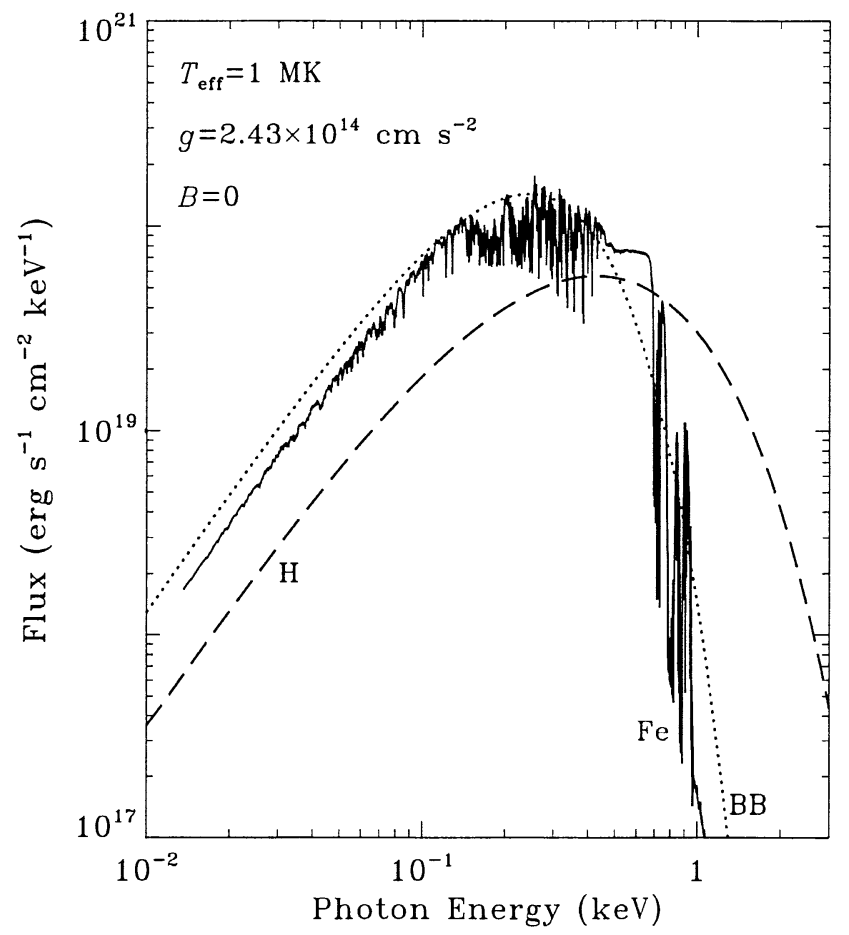

Figure 1. Spectra of hydrogen and iron NS atmospheres, together with the blackbody spectrum, at $T_{\text {eff }}=1 \mathrm{MK}$.

hotter layers. As a result, fitting observed spectra with the standard blackbody model yields spectral (blackbody) temperatures exceeding the true effective temperatures by a factor of $1.5-3$, which makes a great difference for the comparison with the models of NS cooling.

The spectra emitted from iron atmospheres are much more complex due to numerous spectral features produced by iron ions in various stages of ionization (Figure 1). Some of these features are observable even with moderate-resolution (e.g, CCD) spectrometers. On the other hand, when observed with very low energy resolution, the iron atmosphere spectra look very similar to the blackbody spectra. The local specific intensity of radiation increases with the angle between the NS surface and the wave vector, and the shape of the angular distribution depends on photon energy and chemical composition. This (limb-darkening) effect must be taken into account for the fitting of both the spectra and the pulse profiles if the radiation is emitted from hot spots on the NS surface, like in millisecond pulsars (Zavlin \& Pavlov 1998).

\subsection{High-field Neutron Star Atmospheres}

The first models of magnetic hydrogen atmospheres with $B \sim 10^{11}-10^{13} \mathrm{G}$ have been constructed recently (Shibanov et al. 1992; Pavlov et al. 1994, 1995; Za- 


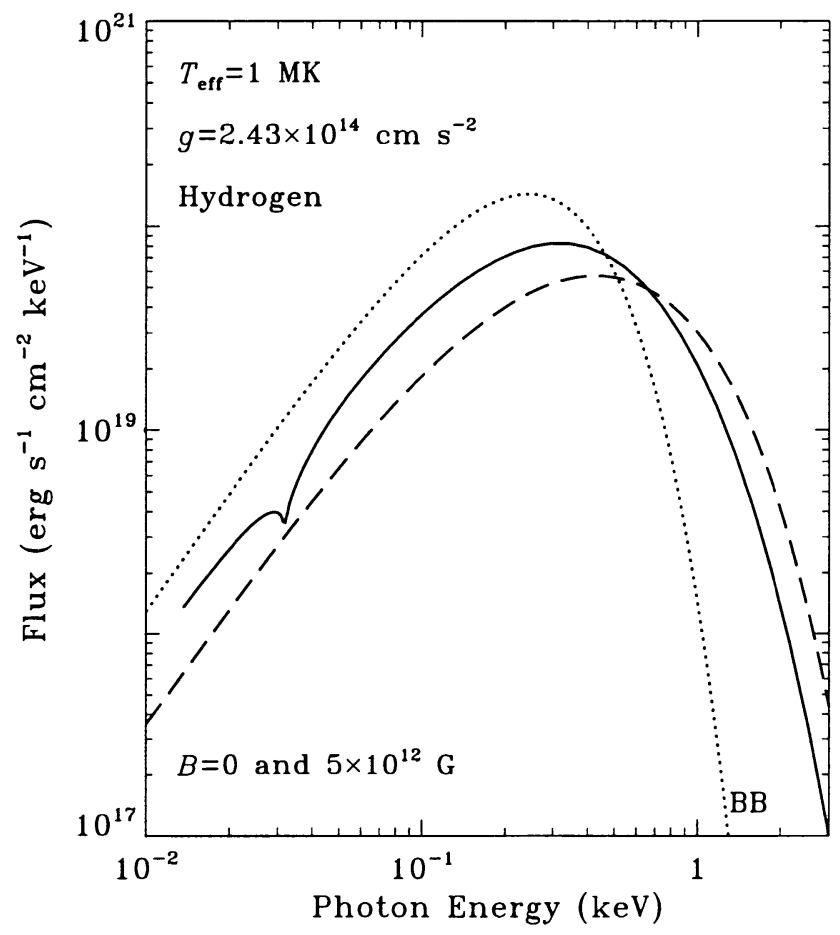

Figure 2. Spectra of high-field and low-field hydrogen atmospheres.

vlin et al. 1995a). These models are based upon simplified opacities of strongly magnetized, partially ionized hydrogen plasma. These opacities do not include the bound-bound transitions, neglect the motional Stark effect, and use a simplified model for ionization equilibrium. Nevertheless, the models provide a qualitatively correct description for the magnetic effects on the emergent radiation, and they are accurate enough in the case of high effective temperatures, $z 1 \mathrm{MK}$, when the hydrogen is almost completely ionized even in the very strong magnetic fields.

Since the magnetic atmospheres are much more transparent in the "extraordinary" polarization mode, for which the opacity is strongly reduced by the magnetic field, very deep (hot and dense) layers are responsible for the observed radiation. Their X-ray spectra are harder than the blackbody spectrum at the same effective temperature, although not as much as the low-field spectra. The only spectral line in the spectra of completely ionized hydrogen atmospheres is the proton cyclotron line at the energy $E_{c p}=\left(m_{e} / m_{p}\right) E_{c e}=6.3\left(B / 1 \times 10^{12} \mathrm{G}\right) \mathrm{eV}$ (see Figure 2). The spectra depend not only on the strength, but also on the direction of the magnetic field, which means that the radiative flux emitted by a rotating NS is pulsed even if the effective temperature is uniform along the NS surface. Angular distribution of the local intensity shows a sharp peak along the magnetic field and a broader peak at intermediate angles (the "pencil" and "fan" components); the widths of the peaks depend on photon energy. This means that 
the pulse profiles of radiation emitted from hot polar caps may be much sharper than those emitted from low-field atmospheres. The pulse shape strongly depends on the mass-to-radius ratio due to bending of photon trajectories in the strong gravitational fields (Shibanov et al. 1995). The radiation emitted from magnetic atmospheres is strongly polarized; the degree of polarization depends on $E, B$, and $M / R$ (Pavlov \& Zavlin 2000).

The first results obtained with the improved hydrogen-atmosphere models (Pavlov \& Zavlin, in preparation) which include the bound-bound transitions show that spectral lines, considerably broadened by the motional Stark effect (Pavlov \& Potekhin 1995), become prominent at $T_{\text {eff }} \lesssim 0.5 \mathrm{MK}$. The strongest line is observed at $E \sim 0.1 \ln \left(B / 5 \times 10^{12} \mathrm{G}\right) \mathrm{keV}$.

Magnetic iron-atmosphere models have been considered by Rajagopal, Romani, \& Miller (1997). Making use of the adiabatic approximation $(\ln \beta \gg 1)$, these authors calculated the energies and wave functions of the iron ions and the radiative opacities of the polarization modes. Although these models are inevitably rather crude, they provide a baseline for comparison with the magnetic hydrogen-atmosphere models and for future work on heavy-element atmosphere modeling. Similarly to the low-field case, the magnetic iron-atmosphere spectra are fairly close to the blackbody spectra when observed with low-resolution detectors. Developing more accurate iron-atmosphere models is important for the adequate interpretation of future high-resolution X-ray observations of NS thermal radiation.

\section{Observational Implications}

To obtain the model radiative flux directly comparable with observational data, the local atmosphere spectral intensities are to be integrated over the visible NS surface with allowance for the gravitational redshift and bending of photon trajectories near the NS (Zavlin, Shibanov, \& Pavlov 1995b). Folded with a detector response, this flux can be used for fitting the observed count rate spectra and pulse profiles. A few examples of such analysis are briefly described below.

\subsection{X-ray Observations}

The low-field models have been successfully applied to interpret the soft X-ray observations of the nearest $(d=180 \mathrm{pc})$, low-field $\left(B \sim 3 \times 10^{8} \mathrm{G}\right)$ millisecond pulsar J0437-4715 (Zavlin \& Pavlov 1998 and references therein). Fitting the atmosphere models to the X-ray spectra and light curves detected with the ROSAT and EUVE missions shows that the observed radiation can be interpreted as emission from two polar caps, with $T_{\text {eff }} \simeq 1 \mathrm{MK}$ and radii $\simeq 1 \mathrm{~km}$, covered with hydrogen or helium. The inferred interstellar hydrogen column density towards the NS, $n_{\mathrm{H}} \simeq(1-3) \times 10^{19} \mathrm{~cm}^{-2}$, agrees with independent estimates obtained from UV observations of stars close to the pulsar. The analysis of the light curves with allowance for bending of photon trajectories allowed us to constrain the mass-to-radius ratio: $1.4<\left(M / M_{\odot}\right)(10 \mathrm{~km} / R)<1.6$ (Pavlov \& Zavlin 1997).

The analysis of the ROSAT spectrum of the middle-aged pulsar B0833-45 in terms of the magnetic hydrogen-atmosphere models (Page, Shibanov, \& Zavlin 1996) yields $n_{\mathrm{H}} \simeq 2 \times 10^{20} \mathrm{~cm}^{-2}$ and the distance of $\simeq 300 \mathrm{pc}$ (for $R=10 \mathrm{~km}$ ), 
in fair agreement with other independent estimates (Cha, Sembach, \& Danks 1999). The obtained temperature, $T_{\text {eff }}=0.7-1.1 \mathrm{MK}$, is, by a factor of 2.5 , lower than the blackbody temperature.

The X-ray spectra detected with the ROSAT and ASCA missions from two radio-silent NSs in supernova remnants, 1E 1207.4-5209 in PKS 1209-52 and RX J0822-4300 in Puppis A, have been successfully fitted with the hydrogen NS atmosphere models (Zavlin, Pavlov, \& Trümper 1998; Zavlin et al. 1999). These fits yield more realistic hydrogen column densities and NS parameters than the blackbody fits. For example, for $1 \mathrm{E} 1207.4-5209$, the atmosphere fit gives $T_{\text {eff }}=1.4-1.9 \mathrm{MK}$ and $d=1.6-3.3 \mathrm{kpc}$ (for $M=1.4 M_{\odot}$ and $R=10 \mathrm{~km}$ ), versus $T=4.2-4.6 \mathrm{MK}$ and $d=11-13 \mathrm{kpc}$ for the blackbody fit. The distances inferred from the atmosphere fits are compatible with those estimated from radio observations of the remnants.

The effective temperatures inferred from fitting the soft X-ray spectra of middle-aged pulsars and radio-quiet NSs in supernova remnants with magnetic $\mathrm{H}$-atmospheres agree with modern NS cooling theories, contrary to the temperatures obtained from the blackbody fits. The best agreement was achieved for the "standard" cooling models with allowance for neutron and proton superfluidity. From the comparison of the model cooling curves with the inferred temperatures, Yakovlev et al. (1999) estimated the following critical superfluidity temperatures for neutrons and protons: $T_{\mathrm{cn}}=200 \mathrm{MK}, T_{\mathrm{cp}}=130 \mathrm{MK}$.

The spectrum of the compact central object of the Cassiopeia A supernova remnant, discovered in the first-light Chandra observation (Tananbaum 1999), can be interpreted (Pavlov et al. 2000) as emission from a strongly magnetized NS with magnetically confined hydrogen or helium polar caps $\left(T_{\mathrm{pc}}^{\infty} \approx 2.8 \mathrm{MK}\right.$, $\left.R_{\mathrm{pc}} \approx 1 \mathrm{~km}\right)$ on a cooler iron surface $\left(T_{\mathrm{s}}^{\infty} \approx 1.7 \mathrm{MK}\right)$. Such temperatures are consistent with the standard models of NS cooling.

The faint X-ray radiation from old pulsars (e.g., B1929+10, B0950+08Wang \& Halpern 1997) is likely emitted from very small $\left(A \sim 100 \mathrm{~m}^{2}\right)$, hot $(T \sim 3 \mathrm{MK})$ regions (polar caps?). This is a challenge to many models of radio pulsars, which predict polar cap luminosities much larger than observed.

\subsection{UV-Optical Observations}

The capabilities of modern optical telescopes, particularly, the Hubble Space Telescope (HST), has opened a new window for studying thermal and nonthermal radiation from NSs. First results can be briefly outlined as follows.

Infrared-optical radiation from middle-aged pulsars (B0833-45, B0656+14, Geminga, B1055-52) is nonthermal; thermal radiation from these objects should dominate in the UV range (Pavlov et al. 1997; Mignani et al. 1998; Harlow, Pavlov, \& Halpern 1998; Golden \& Shearer 1999). Future observations with the HST/STIS would allow us to separate the thermal component and to measure the surface temperatures with high precision.

Optical-UV radiation from old pulsars $(\mathrm{B} 1929+10$, B0950+08) is apparently thermal, although more observations are needed to confirm this conclusion. The temperatures inferred $(T \sim 0.1 \mathrm{MK}-$ Pavlov et al. 1996a) allow one to constrain heating mechanisms presumably operating in old NSs (Larson \& Link 1999; Miralles, Urpin, \& Konenkov 1998). 
Optical-UV radiation from radio-silent, isolated NSs (RX J1856-3754Walter \& Matthews 1997; RX J0720-3125-Motch \& Haberl 1998; Kulkarni \& van Kerkwijk 1998) is almost certainly thermal. The joint analysis of the $\mathrm{X}$-ray/optical data may provide a clue to the nature of these objects, including chemical composition of their surface layers (Pavlov et al. 1996b). The multiwavelength analysis is based upon the result that the spectra of NS atmospheres of different chemical compositions, which can be equally well fitted to the observed X-ray spectrum, correspond to quite different fluxes in the opticalUV band. For instance, the values of the optical flux measured by Walter \& Matthews (1997) rule out the hypothesis that the radiation of RX J1856-3754 is emitted from a low-field hydrogen atmosphere. This method can also be applied to other nearby isolated NSs.

\section{Future Work}

\subsection{Theory}

Although substantial progress has been made in modeling of NS atmospheres, a number of problems must be solved for successful applications of the atmosphere models to the interpretation of observations of a broad class of isolated NSs.

To construct models of the hydrogen atmospheres with superstrong magnetic fields, $B \gtrsim 10^{14} \mathrm{G}$, apparently found in anomalous X-ray pulsars and soft gamma-ray repeaters, investigations of the structure of hydrogen atoms, molecules, and molecular chains in such fields should be carried out (see Lai \& Salpeter 1997 for first results), as well as radiative transitions in these species.

Atoms and ions of heavier elements in strong magnetic fields must be investigated (Pavlov 1998), including the effects of atomic motion (Pavlov \& Mèszàros 1993). So far, only one-electron ions have been studied (Bezchastnov, Pavlov, \& Ventura 1998). Radiative opacities of nonideal plasmas and condensed matter must be calculated.

Magnetized NS atmosphere models of different chemical compositions are needed. In particular, magnetized helium atmospheres should be modeled and compared with observations.

\subsection{Observations}

Observations with the Chandra and XMM X-ray observatories provide much higher energy resolution ( $E / \Delta E$ up to 1000$)$ than that achieved with the previous X-ray missions, which would allow one to detect atmospheric spectral lines and photoionization jumps. From the analysis of these features, it would be possible to determine chemical composition, magnetic fields, and mass-to-radius ratios for several middle-aged pulsars and radio-quiet NSs in SNRs.

Observations with the Chandra X-ray observatory provide very high spatial resolution, $\lesssim 1^{\prime \prime}$, which is especially important for separation of the point source radiation from the diffuse radiation in supernova remnants. The importance of high resolution has been demonstrated by the detection of the compact central object of the Cas A supernova remnant (see Section 3.1).

We expect very interesting results from observations of nearby pulsars and NSs with the HST/STIS/MAMA detectors. These observations would allow us 
to separate out the thermal component in radiation of middle-aged pulsars based on the UV-optical spectra and pulse shapes, to measure the temperature, and to infer the chemical composition of NS atmospheres.

Acknowledgments. This work was partially supported by NASA grants NAG5-6907 and NAG5-7017.

\section{References}

Becker, W., \& Trümper, J. 1997, A\&A, 326, 682

Bezchastnov, V. G., Pavlov, G. G., \& Ventura, J. 1998, Phys. Rev. A, 58, 180

Brown, E. F., Bildsten, L., \& Rutledge, R. E. 1998, ApJ, 504, L95

Bulik, T., \& Pavlov, G. G. 1996, ApJ, 469, 373

Caraveo, P. A., Bignami, G. F., \& Trümper, J. 1996, ARA\&A, 7, 209

Cha, A. N., Sembach, K. R., \& Danks, A. C. 1999, ApJ, 515, L25

Gnedin, Y. N., \& Pavlov, G. G. 1974, Sov. Phys.-JETP, 38, 903

Golden, A., \& Shearer, A. 1999, A\&A, 342, L5

Greenstein, G., \& Hartke, G. J. 1983, ApJ, 271, 283

Halpern, J. P., \& Wang, F. Y.-H. 1997, ApJ, 477, 905

Harlow, J. J. B., Pavlov, G. G., \& Halpern, J. 1998, BAAS Vol. 30, No. 4, AAS Meeting 193, \#41.07

Iglesias, C. A., \& Rogers, F. J. 1996, ApJ, 464, 943

Kulkarni, S., \& van Kerkwijk, M. 1998, ApJ, 507, L49

Lai, D., \& Salpeter, E. 1997, ApJ, 491, 270

Larson, M. B., \& Link, B. 1999, ApJ, 521, 271

Mignani, R., Caraveo, P. A., \& Bignami, G. F. 1998, Adv. Space Res., 21, 197

Miralles, J. A., Urpin, V., \& Konenkov, D. 1998, ApJ, 503, 368

Motch, C., \& Haberl, F. 1998, A\&A, 333, 59

Ögelman, H. 1995, in The Lives of the Neutron Stars, eds. M. A. Alpar, Ü. Kiziloğlu, \& J. van Paradijs (Dordrecht: Kluwer), 101

Page, D., Shibanov, Y. A., \& Zavlin, V. E. 1996, in MPE Reports, 263, Röntgenstrahlung from the Universe, eds. H.-U. Zimmerman, J. Trümper, \& H. Yorke (Garching: MPE), 173

Pavlov, G. G. 1998, in Atoms and Molecules in Strong External Fields, eds. P. Schmelcher \& W. Schweizer (New York: Plenum), 37

Pavlov, G. G., \& Mèszàros, P. 1993, ApJ, 416, 752

Pavlov, G. G., \& Potekhin, Y. A. 1995, ApJ, 450, 883

Pavlov, G. G., \& Shibanov, Y. A. 1978, Soviet Ast., 22, 43

Pavlov, G. G., Shibanov, Y. A., Ventura, J., \& Zavlin, V. E. 1994, A\&A, 289, 847

Pavlov, G. G., Shibanov, Y. A., Zavlin, V. E., \& Meyer, R. D. 1995, in The Lives of the Neutron Stars, eds. M. A. Alpar, Ü. Kiziloğlu \& J. van Paradijs (Dordrecht: Kluwer), 71

Pavlov, G. G., Stringfellow, G. S., \& Córdova, F. A. 1996a, ApJ, 467, 370 
Pavlov, G. G., Welty, A. D., \& Córdova, F. A. 1997, ApJ, 489, L75

Pavlov, G. G., \& Zavlin, V. E. 1997, ApJ, 490, L91

Pavlov, G. G., \& Zavlin, V. E. 2000, ApJ, 529, in press

Pavlov, G. G., \& Zavlin, V. E., in preparation

Pavlov, G. G., Zavlin, V. E., Aschenbach, B., Trümper, J., \& Sanwal, D. 2000, ApJ, in press

Pavlov, G. G., Zavlin, V. E., Trümper, J., \& Neuhäuser, R. 1996b, ApJ, 472, L33

Pethick, C. J. 1992, Rev. Mod. Phys., 64, 1133

Rajagopal, M., \& Romani, R. W. 1996, ApJ, 461, 327

Rajagopal, M., Romani, R. W., \& Miller, M. C. 1997, ApJ, 479, 347

Romani, R. W. 1987, ApJ, 313, 718

Rutledge, R. E., Bildsten, L., Brown, E. F., Pavlov, G. G, \& Zavlin, V. E. 1999, ApJ, 514, 945

Shibanov, Y. A., Pavlov, G. G., Zavlin, V. E., Qin, L., \& Tsuruta, S. 1995, in Ann. NY Acad. Sci., 759, Proc. 17th Texas Symp. on Relativistic Astrophysics, eds. H. Bohringer, G. Morfill, \& J. Trümper (New York: ANYAS), 291

Shibanov, Y. A., \& Yakovlev, D. G. 1996, A\&A, 309, 171

Shibanov, Y. A., Zavlin, V. E., Pavlov, G. G., \& Ventura, J. 1992, A\&A, 266, 313

Tananbaum, H. 1999, IAU Circ. 7246

Tsuruta, S. 1998, Phys. Rep., 292, 1

Van Riper, K. A., Link, B., \& Epstein, R. 1995, ApJ, 448, 294

Walter, F. M., \& Matthews, L. M. 1997, Nature, 389, 358

Wang, F. Y.-H., \& Halpern, J. P. 1997, ApJ, 482, L159

Yakovlev, D. G., Levenfish, K. P., \& Shibanov, Y. A. 1999, Physics-Uspekhi, 169,825

Yancopoulos, S., Hamilton, T. T., \& Helfand, D. J. 1994, ApJ, 429, 832

Zavlin, V. E., \& Pavlov, G. G. 1998, A\&A, 329, 583

Zavlin, V. E., \& Pavlov, G. G. 2000, ApJ, submitted

Zavlin, V. E., Pavlov, G. G., \& Shibanov, Y. A. 1996, A\&A, 315, 141

Zavlin, V. E., Pavlov, G. G., Shibanov, Y. A., \& Ventura, J. 1995a, A\&A, 297, 441

Zavlin, V. E., Pavlov, G. G., \& Trümper, J. 1998, A\&A, 331, 821

Zavlin, V. E., Shibanov, Y. A., \& Pavlov, G. G. 1995b, Astr. Lett., 21, 149

Zavlin, V. E., Trümper, J., \& Pavlov, G. G. 1999, ApJ, 525, 959 